\title{
Mapping and analysis of a novel candidate Fusarium wilt resistance gene FOC1 in Brassica oleracea
}

Honghao Lv', Zhiyuan Fang ${ }^{1}$, Limei Yang ${ }^{1}$, Yangyong Zhang ${ }^{1}$, Qingbiao Wang ${ }^{2}$, Yumei Liu' ${ }^{1}$ Mu Zhuang ${ }^{1}$, Yuhong Yang ${ }^{1}$, Bingyan Xie ${ }^{1}$, Bo Liu', Jisheng Liu', Jungen Kang ${ }^{2^{*}}$ and Xiaowu Wang ${ }^{1 *}$

\begin{abstract}
Background: Cabbage Fusarium wilt is a major disease worldwide that can cause severe yield loss in cabbage (Brassica olerecea). Although markers linked to the resistance gene FOC1 have been identified, no candidate gene for it has been determined so far. In this study, we report the fine mapping and analysis of a candidate gene for FOC1 using a double haploid (DH) population with 160 lines and a $F_{2}$ population of 4000 individuals derived from the same parental lines.

Results: We confirmed that the resistance to Fusarium wilt was controlled by a single dominant gene based on the resistance segregation ratio of the two populations. Using InDel primers designed from whole-genome re-sequencing data for the two parental lines (the resistant inbred-line 99-77 and the highly susceptible line 99-91) and the DH population, we mapped the resistance gene to a 382-kb genomic region on chromosome C06. Using the $F_{2}$ population, we narrowed the region to an 84-kb interval that harbored ten genes, including four probable resistance genes (R genes): Bol037156, Bol037157, Bol037158 and Bol037161 according to the gene annotations from BRAD, the genomic database for $B$. oleracea. After correcting the model of the these genes, we re-predicted two $R$ genes in the target region: re-Bol037156 and re-Bol0371578. The latter was excluded after we compared the two genes' sequences between ten resistant materials and ten susceptible materials. For re-Bol037156, we found high identity among the sequences of the resistant lines, while among the susceptible lines, there were two types of InDels (a 1-bp insertion and a 10-bp deletion), each of which caused a frameshift and terminating mutation in the cDNA sequences. Further sequence analysis of the two InDel loci from 80 lines (40 resistant and 40 susceptible) also showed that all $40 \mathrm{R}$ lines had no InDel mutation while 39 out of $40 \mathrm{~S}$ lines matched the two types of loci. Thus re-Bol037156 was identified as a likely candidate gene for $\mathrm{FOC1}$ in cabbage.
\end{abstract}

Conclusions: This work may lay the foundation for marker-assisted selection as well as for further function analysis of the FOC1 gene.

Keywords: Brassica oleracea, Fusarium wilt, Resistance gene, FOC1, Map-based cloning

\footnotetext{
* Correspondence: wangxiaowu@caas.cn; kangjungen@nercv.org

${ }^{1}$ Institute of Vegetables and Flowers, Chinese Academy of Agricultural Sciences, Key Laboratory of Biology and Genetic Improvement of Horticultural Crops, Ministry of Agriculture, 12\#Zhongguancun Nandajie Street, Beijing 100081, China

${ }^{2}$ Beijing Vegetable Research Center, Beijing Academy of Agriculture and Forestry Sciences, Key Laboratory of Biology and Genetic Improvement of Horticultural Crops (North China), Ministry of Agriculture, 50\# Zhanghua Street, Beijing 100097, China
} 


\section{Background}

Cabbage (Brassica oleracea L. var. capitata L.) is one of the most cultivated vegetables worldwide. In 2010, more than two million hectares were estimated to be under production, with an average yield of 27.8 tonnes per hectare (FAOSTAT 2010). Cabbage Fusarium wilt (CFW) is a destructive fungal disease caused by Fusarium oxysporum f. sp. conglutinans, which for many years has caused severe losses to cabbage yields all over the world. CFW was first discovered in the USA in 1899, and then in Japan and several other countries in the following decades $[1,2]$. In recent years, CFW was discovered in several provinces in China and has spread rapidly [3-5]. It is a typical soil-borne disease that is hard to control by traditional methods such as chemicals and crop rotation. The most effective control has been the use of resistant cabbage varieties [6-8]. Therefore, the development and use of new resources have become the main goals in current resistance breeding programs for cabbage.

It has been reported that two races in the formae speciale conglutinans could infect cabbage $[9,10]$. Race 1 was found in several countries, and the inheritance of cabbage resistance to it was determined to be of the single dominant type $[11,12]$. Race 2 was found only in the US and Russia, and the mode of inheritance of resistance to it remains unclear $[13,14]$.

Although CFW is a major disease for cabbage, not enough attention has been paid to it, and research on CFW is still at a preliminary stage. Several molecular markers have been developed for the FOC gene $[15,16]$, but, until now, no reports have focused on gene analysis or on functional studies of candidate genes. In a previous study, we located the resistance gene on chromosome C06 of cabbage, with two InDel markers flanking the gene at 1.2 and $0.6 \mathrm{cM}$ [17]. Currently, the large scale re-sequencing of plant genomes has allowed for the application of insertion/ deletion (InDel) and single nucleotide polymorphism (SNP) markers. In addition, the $B$. oleracea genome sequence has recently been published [18]. This has significantly advanced map-based cloning in cabbage, which was originally labor-intensive. InDel markers can be obtained easily, either using bioinformatic methods or from reliable visualization of alleles of different size [19].

The objectives of the current study were (i) to fine map the FOC1 gene using one double haploid (DH) and one $\mathrm{F}_{2}$ population, and newly developed InDel markers designed from the whole-genome re-sequencing data of the two parental lines (the resistant inbred-line 99-77 and the highly susceptible line 99-91, and (ii) to analyze the genes that fell into the candidate genomic region, and finally identify the candidate gene.

\section{Results}

Resistance segregation of the $\mathrm{DH}$ and $\mathrm{F}_{2}$ populations

We obtained a total of $160 \mathrm{DH}$ lines through microspore culture [20] and over $4000 \mathrm{~F}_{2}$ individuals derived from the same parental lines through hand pollination. Resistance segregation ratios in the $\mathrm{DH}$ and $\mathrm{F}_{2}$ populations were evaluated using the inoculation test. The result indicated that in the DH population, 68 lines showed immunity to Fusarium wilt while 92 were highly susceptible. The $\chi^{2}$ goodness-of-fit test indicated that the segregation ratio fitted a 1:1 ratio in the $\mathrm{DH}$ population. Of the 4000 individuals in the $F_{2}$ population, 2964 plants were classified as resistant and 1012 were classified as susceptible; 24 individuals were excluded because of an unclear resistance performance. The $\chi^{2}$ goodness-of-fit test fitted a 3:1 Fusarium wilt resistance segregation ratio. These results indicated that the resistance in the two populations was controlled by a single dominant gene, which was consistent with the results from our prior study [5].

\section{Mapping of FOC1 using the $\mathrm{DH}$ and $\mathrm{F}_{2}$ populations}

In an earlier study, we located the candidate gene in a $1.8 \mathrm{cM}$ genomic region on chromosome C06, with two InDel markers, M10 and A1, flanking FOC1 at $1.2 \mathrm{cM}$ and $0.6 \mathrm{cM}$ respectively [17]. Based on this result, we designed additional InDel primers in this region to map the FOC1 gene more precisely (Table 1). In addition, we obtained two pairs of simple sequence repeat (SSR) primers, R3 and R7, described in a previous study in which the candidate gene was mapped to a linkage group with two markers flanking the gene at $4.6 \mathrm{cM}$ and $1.2 \mathrm{cM}$ [16]. A total of 18 markers were anchored to the genetic map using JoinMap 4.0, which matched to the physical map according to the positions of the markers (Figure 1a). Finally, using these markers and the $\mathrm{DH}$ population, we mapped the FOC1 gene to a 382-kb genomic region between the S3 and A1 markers (Figure 1b). Moreover, the SSR markers indicated that this was the same region as the region reported in the previous study (Figure 1b) [16].

The flanking markers A1 and S3 obtained from the $\mathrm{DH}$ population were used to screen the $\mathrm{F}_{2}$ population for recombinant individuals, which were then evaluated for their resistance to Fusarium wilt. Next, more markers between $\mathrm{A} 1$ and $\mathrm{S} 3$ were used to genotype these individuals. As a result, the FOC1 gene was narrowed to an 84-kb genomic region with V17 and S9 as the flanking InDel markers (Figure 1c). Both InDel markers had two recombinants with FOC1.

\section{Candidate gene analysis}

From BRAD (http://brassicadb.org) [21], the genomic database for $B$. oleracea where the reference genome 
Table 1 Primers used for fine mapping of FOC1

\begin{tabular}{|c|c|c|c|c|}
\hline Primer & Physical position on $\mathrm{C06}$ & Type & $\mathrm{Tm}$ & Sequences $\left(5^{\prime}-3^{\prime}\right)$ \\
\hline R7 & 38515717 & SSR & $58^{\circ} \mathrm{C}$ & TCACTCCTCTCGCAGATTCA/TGGAATCGCTITAAGCAGATGC \\
\hline M10 & 38577450 & InDel & $55^{\circ} \mathrm{C}$ & CACTTGCTCCAGTTTCTGTA//AACTATGGATAAAAGGCGTG \\
\hline S3 & 38605567 & InDel & $55^{\circ} \mathrm{C}$ & TाTCGGTTGACAGAGAAAGT//AGGAGAGAATCAAAAGCTCC \\
\hline S4 & 38610913 & InDel & $55^{\circ} \mathrm{C}$ & GGACTCACCTITITGTGTGT//AGATTACCCGTTTTCTCCTC \\
\hline S6 & 38645784 & InDel & $55^{\circ} \mathrm{C}$ & GGACTCCTTTGATCCTCTCT//GAATGCAACCATGTAAACAG \\
\hline S7 & 38685934 & InDel & $55^{\circ} \mathrm{C}$ & ACTTGACAGCTTTCCATCTG//TTTCTGCCCAGGTACTTAAA \\
\hline V17 & 38743868 & InDel & $55^{\circ} \mathrm{C}$ & GCAGATAATAATCCACACGTC//TACCACCTTTITCTGGCTTA \\
\hline s9 & 38827074 & InDel & $55^{\circ} \mathrm{C}$ & CTGAAACTCGGGAATACATC//AAGCTTCCCACACTCTCTCT \\
\hline V18 & 38828622 & InDel & $55^{\circ} \mathrm{C}$ & TACAAGCTCCTTGTGGATTT//CAATCCTTGGCTGTITTAAG \\
\hline S12 & 38986625 & InDel & $55^{\circ} \mathrm{C}$ & GCCCTCATTACGTTTAACAA//GAGTTGCAGCCTTCAAGTAT \\
\hline A1 & 38988089 & InDel & $55^{\circ} \mathrm{C}$ & TGACATAACCACTAGGAGCA//GCAGAAGCTTTGATGAAGTT \\
\hline $\mathrm{S} 13$ & 39018573 & InDel & $55^{\circ} \mathrm{C}$ & TGATGGACTGAAACCTAACC//AGGAGGATGAGGAAGAACC \\
\hline V23 & 39029152 & InDel & $55^{\circ} \mathrm{C}$ & СTCTITATTGCAACGACACA/ICAATACGTCACTCATCACG \\
\hline A2 & 39107094 & InDel & $55^{\circ} \mathrm{C}$ & TGGTCTTGGTCTCTTGTTI//AGCATTAACACTGACACCCT \\
\hline $\mathrm{A} 12$ & 41310866 & InDel & $55^{\circ} \mathrm{C}$ & GCGAACCGACCCTAGTAT//CAAAGCCAAGAAGGTGTTA \\
\hline A15 & 41982164 & InDel & $55^{\circ} \mathrm{C}$ & GGTTTGAACCTCTGAAAATG//TGTGATTTCGGTTAGAGTCC \\
\hline R3 & 42957515 & SSR & $58^{\circ} \mathrm{C}$ & CGCTATGGATAATGTGTTCA//ATTAACAGCGAGGATAGCAA \\
\hline
\end{tabular}

sequence for B. oleracea is now available [18], we obtained the reference genome sequence and found that there were ten annotated genes in the 84-kb genomic region (Figure 1d, Table 2). However, we noticed that there was no gene numbered Bol037164 in the database, and the prediction of the sequence between Bol037163 and Bol037165 showed that no gene existed here. The best hits of the ten annotated B. oleracea genes to the Brassica rapa and Arabidopsis thaliana genome are shown in Table 2. Annotation analysis of these genes from BRAD indicated that four of them were most likely related to disease resistance [22]: Bol037161 was predicted to have transcription factor activity; Bol037156, Bol037157 and Bol037158 contained the most commonly conserved domain of $\mathrm{R}$ proteins: leucine-rich repeat (LRR), toll-interleukin receptor (TIR) domain and LRR domain respectively (Table 2), based on the InterPro domain annotation and Gene Ontology annotation for $B$. olerecea and $A$. thaliana obtained from BRAD.

Primers were designed to amplify the full length of the four candidate genes in ten resistant inbred lines (including the resistant parental line) and ten susceptible inbred lines (including the susceptible parental line) (Table 3). However, no common sequence variation was found between the R_bulk and the S_bulk for all the four genes.

Nevertheless, all the three genes seemed too short with only one $\mathrm{R}$ protein domain based on a common sense of TIR-NBS-LRR or NBS-LRR type resistance gene ( $R$ gene) except Bol037161 as a transcription factor (Table 2)
[22]. Thus comparative genomics analysis was made between $B$. rapa genes and $B$. oleracea ones in the target region (Figure $2 \mathrm{a}, \mathrm{b}$ and $\mathrm{c}$ ). The results showed that the homologous gene for Bol037156 in B. rapa was a putative TIR-NBS-LRR type R gene Bra012688, or BrNL17 [GenBank: FJ842771.1] (http://blast.ncbi.nlm.nih.gov) [23], while the homologous gene for Bol037157 and Bol037158 was also a putative TIR-NBS-LRR type $\mathrm{R}$ gene Bra012689, or BrTNL32 [GenBank: FJ842772.1] (Figure $2 \mathrm{~b}$ and $\mathrm{c}$ ). To further analyze these genes, the target genomic sequence of $B$. olerecea containing the three genes was submitted to the FGENSH program (http://linux1.softberry.com/berry.phtml) [24] to conduct gene prediction. Because the reference line was highly susceptible in our inoculation test which might affect the prediction result, the re-prediction program used the target sequence of the resistant parental line $\mathrm{P}_{1}$.

The results indicated that the re-predicted gene Bol037156, designated as re-Bol037156, was a putative TIR-NBS-LRR gene with high identity to Bra012688, while the other two genes Bol037157 and Bol037158 were re-predicted to be another putative TIR-NBS-LRR gene, named re-Bol0371578 which showed high identity to Bra012689 (Figure 2d).

The cDNA sequences showed high identity to the repredicted genes' sequences (Figure 2e). The B. olerecea transcriptome reads mapping results further confirmed the re-prediction result (Figure 2f). Thus, we obtained two re-predicted $B$. oleracea genes as the candidates, both of which were putative TIR-NBS-LRR type $R$ genes. 


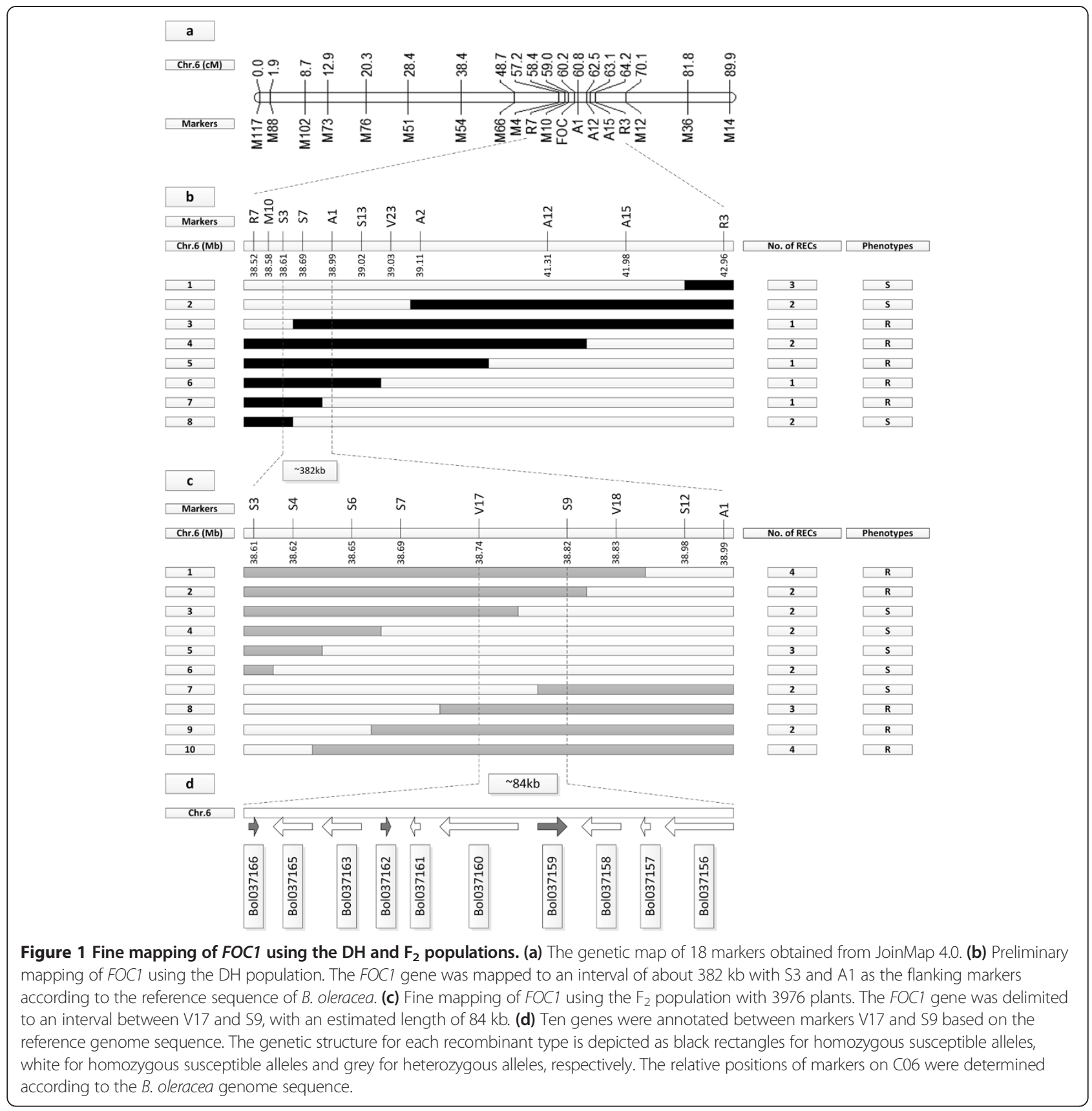

To further identify which one was the most likely candidate gene for CFW resistance, primers were designed to obtain both full length DNA and cDNA of the two genes from ten resistant cabbage inbred lines including the resistant parent (R_bulk) and ten susceptible lines including the susceptible parent (S_bulk) in order to conduct sequence alignment and mutation loci analysis (Table 4). The results showed that no consistent variation was found between the R_bulk and the S_bulk for the gene re-Bol0371578. While for re-Bol037156, the cDNA from the ten R_bulk lines showed a high identity of $98.97 \%$ and no InDel was found. The cDNA from the ten $\mathrm{S}$ _bulk lines also showed high identity. When the R_bulk and S_bulk lines were compared, two types of InDels in the S_bulk were found; one was a 10-bp deletion in two sequences and the other was a 1-bp insertion in the other eight sequences (Figure 3a). Both InDels caused frameshifts in the open reading frames that resulted in terminating mutations in the susceptible lines (Figure 3b).

Thus, the newly predicted gene re-Bol037156 was considered as a candidate gene for CFW resistance, with a total length of $6.4 \mathrm{~kb}$, CDS length of $4.1 \mathrm{~kb}$, eight exons and seven introns (Figure 2e). The candidate gene was 
Table 2 Annotation of the B. oleracea genes in the candidate region

\begin{tabular}{|c|c|c|c|c|c|c|c|}
\hline Bra genes $^{\mathrm{a}}$ & E-value & Bol genes $^{b}$ & Gene position on $\mathrm{CO6}^{\mathrm{C}}$ & Bol IPR annotation $^{d}$ & $A T I^{\mathrm{e}}$ & E-value & $A T$ GO annotation ${ }^{f}$ \\
\hline- & - & Bol037166 & $38746794-38747237$ & DNA polymerase & AT5G18880 & $5 E-21$ & biological process unknown \\
\hline- & - & Bol037165 & $38749215-38750398$ & Alpha/beta hydrolase & AT4G16690 & $8 \mathrm{E}-126$ & hydrolase activity \\
\hline- & - & Bol037163 & 38753393-38754437 & Ribosomal protein & AT4G16720 & $2 \mathrm{E}-107$ & ribosomal protein \\
\hline - & - & Bol037162 & $38754883-38755278$ & Exostosin-like & AT4G16745 & $7 \mathrm{E}-68$ & exostosin family protein \\
\hline- & - & Bol037161 & $38795305-38795850$ & transcriptional factor & AT4G16750 & $2 \mathrm{E}-73$ & transcription factor \\
\hline- & - & Bol037160 & $38801162-38804053$ & oxygenase & AT4G16765 & $1 \mathrm{E}-117$ & oxidoreductase activity \\
\hline Bra012690 & 0 & Bol037159 & $38810947-38812479$ & Lipase & AT4G16820 & 0 & lipase activity \\
\hline Bra012689 & 0 & Bol037158 & $38813370-38815166$ & LRR & AT4G16950 & $2 \mathrm{E}-82$ & RPP5; nucleotide binding \\
\hline Bra012689 & $2 \mathrm{E}-90$ & Bol037157 & $38816186-38816452$ & TIR & AT4G16890 & $1 E-32$ & nucleotide binding \\
\hline Bra012688 & 0 & Bol037156 & $38822022-38824544$ & LRR & AT4G08450 & $2 \mathrm{E}-17$ & disease resistance protein \\
\hline Bra012687 & 0 & Bol037155 & $38831452-38833049$ & Pollen allergen & AT4G17030 & $3 E-117$ & cellulose and wall loosening \\
\hline
\end{tabular}

${ }^{\mathrm{a}} B$. rapa homologous genes in the candidate region.

${ }^{\mathrm{b}}$ Ten $B$. oleracea genes in the candidate region. The likely resistance genes and their information are indicated in bold.

'The physical position of the ten B. oleracea genes on chromosome C06.

dinterPro domain annotations for the ten B. oleracea genes obtained from BRAD.

e The best hits of the ten B.oleracea genes to A.thaliana (AT).

${ }^{f} \mathrm{GO}$ annotations for the ten $B O /$ to $A T$ best-hit genes obtained from BRAD.

predicted to encode a TIR-NBS-LRR type protein containing five conserved domains including one TIR domain, one NBS domain and three LRR domains according to a Pfam sequence search result (Figure 2e) (http://pfam.sanger.ac.uk/) [25].

\section{Validation of the FOC1 gene}

To verify re-Bol037156 as the candidate gene for cabbage Fusarium wilt resistance gene $F O C 1$, primers were designed to obtain the two InDel loci sequence from 30 resistant cabbage inbred lines (R_bulk) and 30 susceptible lines (S_bulk) (Table 5). Together with the sequences of the former 20 lines, we conducted sequence alignment.

The sequences from the $40 \mathrm{R}$ _bulk lines showed high identity and no InDel was found at the two loci. The 40 sequences from the ten S_bulk lines also showed high identity. When the R_bulk and S_bulk lines were compared, two types of InDels in the S_bulk were found as was described above: sequences of 29 lines matched the 1-bp insertion and sequences of other 10 lines matched the 10-bp deletion. However, sequence of one line (S12) in the S_bulk matched neither of the two types of InDels. As we didn't obtain the full length cDNA of the

Table 3 Primers used for cloning full length gene of four candidate genes

\begin{tabular}{lc}
\hline Primers & Sequences $\mathbf{( 5}^{\prime}$-3') \\
\hline Bol037156 & GGTGACACTTCCTTCCTCCA//GCTCCATCGCCATCAAAGTT \\
Bol037157 & TCGATTICCTCACCACCGA/TAAGCATCCCACCTGAGCAA \\
Bol037158 & CTCAGATTGCTCAGGTGGGA/ACACACCCACATTGCGTTAC \\
Bol037161 & GAAACTGTGTCCCTGCCATG//TGTGGCAGAGTTACATGGGT \\
\hline
\end{tabular}

gene in these lines, we concluded that there might be other types of variation that caused loss of gene function. The result of 80 lines' sequences alignment further validated that re-Bol037156 was a most likely candidate for FOC1.

\section{Discussion}

\section{Fusarium wilt and cabbage resistance breeding}

CFW is a soil-borne disease that is hard to control by traditional physical or chemical methods. Therefore, CFW has spread quickly across China from Beijing in 2003 to the Hebei, Gansu, Shanxi, and Shaanxi provinces where it is now present. Thus, the application of resistant varieties is of great significance [6-8]. Indeed, their application has often been cited as a good example of using a plant's own power to overcome fungal disease. Two factors account for this: firstly, the inheritance of cabbage resistance to Race 1 is single dominant $[11,12]$, as was also shown in our prior study [5], and this meant that the $R$ gene could be used easily in the $F_{1}$ progeny; and secondly, although two races in the forma speciale conglutinans can infect cabbage, only Race 1 is found worldwide, while Race 2 has been reported only in the USA and Russia [13,14], indicating that the immutability of the pathogen over decades has also contributed to the successful use of the single dominant $\mathrm{R}$ gene. Because of the significance of this R gene, it is necessary to discover the mechanism by which this $\mathrm{R}$ gene overcomes CFW.

For many years, MAS has promoted the traditional breeding process. However, few studies have reported molecular markers for FOC1. Pu et al. [16] located the $\mathrm{R}$ gene on a linkage group, with two SSR markers flanking the gene at 4.6 and $1.2 \mathrm{cM}$ respectively without 


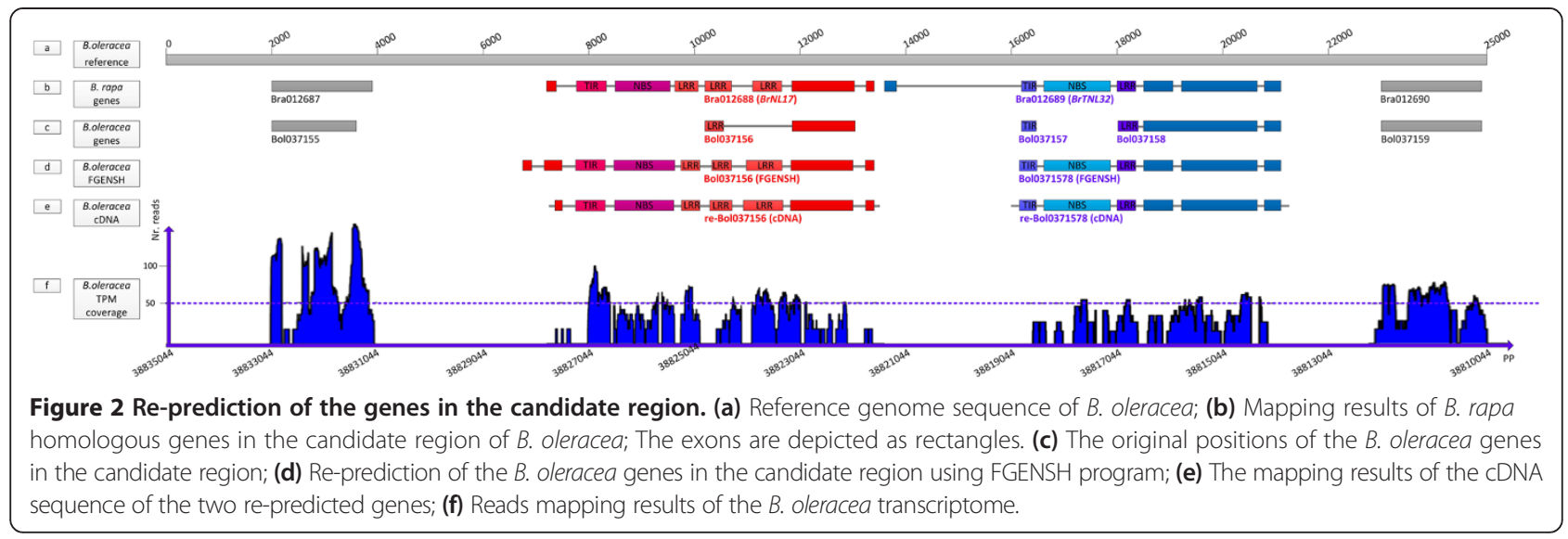

chromosome information, and in a previous study, we located the $\mathrm{R}$ gene on chromosome C06, with two InDel markers flanking the gene at 1.2 and $0.6 \mathrm{cM}$ [17]. In this study, we further refined the map and identified a candidate gene for FOC1. The results reported here will be useful for cabbage breeders because they may facilitate MAS in the breeding process.

\section{Map-based cloning of FOC1 using InDel markers and two populations}

Traditional molecular marker assays are usually laborintensive and time-consuming, and the markers, like SSRs, that are used are frequently of low polymorphism and not reliable. Further, they often produce complicated stripes when the PCR products are isolated in the gels. In recent years, as more and more plant genome sequences become available including $B$. oleracea genome [18], InDel and SNP markers have become the markers of choice because they are generally obtained easily using bioinformatic method and their PCR products are more reliable and easier to detect. In this study, for example, we easily acquired 1000 pairs of InDel primers on one chromosome and although we used only a small number of them, the polymorphic primers accounted for $75 \%$ of all.

In this study, the InDel primers were designed based on the cabbage reference sequence and the whole-

Table 4 Primers used for cloning of re-Bol037156 and re-Bol0371578 in the $R$ _bulk and S_bulk lines

\begin{tabular}{|c|c|}
\hline Primers & Sequences $\left(5^{\prime}-3^{\prime}\right)$ \\
\hline re-Bol037156 (DNA) & $\begin{array}{l}\text { CCCCAGATTCACAGCATTCG// } \\
\text { ATACGAGTGTGTCTTCGCCA }\end{array}$ \\
\hline re-Bol037156 (cDNA) & $\begin{array}{c}\text { ATCTTCACCACTCCAGTCA// } \\
\text { ACTTAACTGGAATGAGCTAACCA }\end{array}$ \\
\hline re-Bol0371578 (DNA) & $\begin{array}{l}\text { TCGATTITCCTCACCACCGA// } \\
\text { ACCCAGCCCTAATGAAAGCT }\end{array}$ \\
\hline re-Bol0371578 (cDNA) & $\begin{array}{l}\text { TGAGTTGGTCCACGTACTTGA// } \\
\text { AGGAGAAAGGGAAAGACGCA }\end{array}$ \\
\hline
\end{tabular}

genome re-sequencing data for the parental lines. Therefore, the polymorphic primers accounted for a relative higher percentage. Also, genome-sequence-based InDel primers allow us to quickly design and add more markers to the target interval on the chromosome in order to narrow the mapping interval. As a result, we successfully mapped the $\mathrm{R}$ gene to chromosome C06 in a time-saving way based on the $\mathrm{DH}$ and $\mathrm{F}_{2}$ populations.

Most characterized $\mathrm{R}$ genes reside in clusters rather than being distributed equally on chromosomes [26]. Here also, the candidate region harbored two TIR-NBSLRR genes: re-Bol037156 and re-Bol0371578.

\section{Plant resistance gene to disease caused by Fusarium oxysporum}

As far as we know, more than $70 \mathrm{R}$ genes have been cloned in the past decades [27]. The main categories of these genes include the coiled coil-nucleotide binding site-leucine rich repeat (CC-NB-LRR) class, the TIRNBS-LRR class, the kinase class, the LRR kinase class and the extracellular LRRs [22]. The most significant TIR-NBS-LRR class $\mathrm{R}$ gene may be the tobacco $N$ gene conferring resistance to tobacco mosaic virus [28]. For $N$ gene, all the three conserved domains are indispensable for a normal function [29].

Until now, the Fusarium wilt $\mathrm{R}$ genes have been cloned in three plant species: Arabidopsis, tomato, and melon [30-32]. The function of the melon $\mathrm{R}$ gene, FOM2, has not yet been established [31]. RFO1 in Arabidopsis was reported to be a wall-associated kinase, which was neither a representative $R$ gene nor race-specific [32]. On the other hand, the CFW $\mathrm{R}$ gene was race-specific and can be overcome by Race 2 [13]. In tomato, I-2 was shown to be a typical $\mathrm{R}$ gene, with the encoded protein belonging to the CC-NBS-LRR type [30]. A low identity of $41 \%$ was found among the three cloned $\mathrm{R}$ genes, indicating that although all three were $\mathrm{R}$ genes to Fusarium wilt, different mechanisms may be involved. We also performed whole-genome blast searches with a cut-off value 


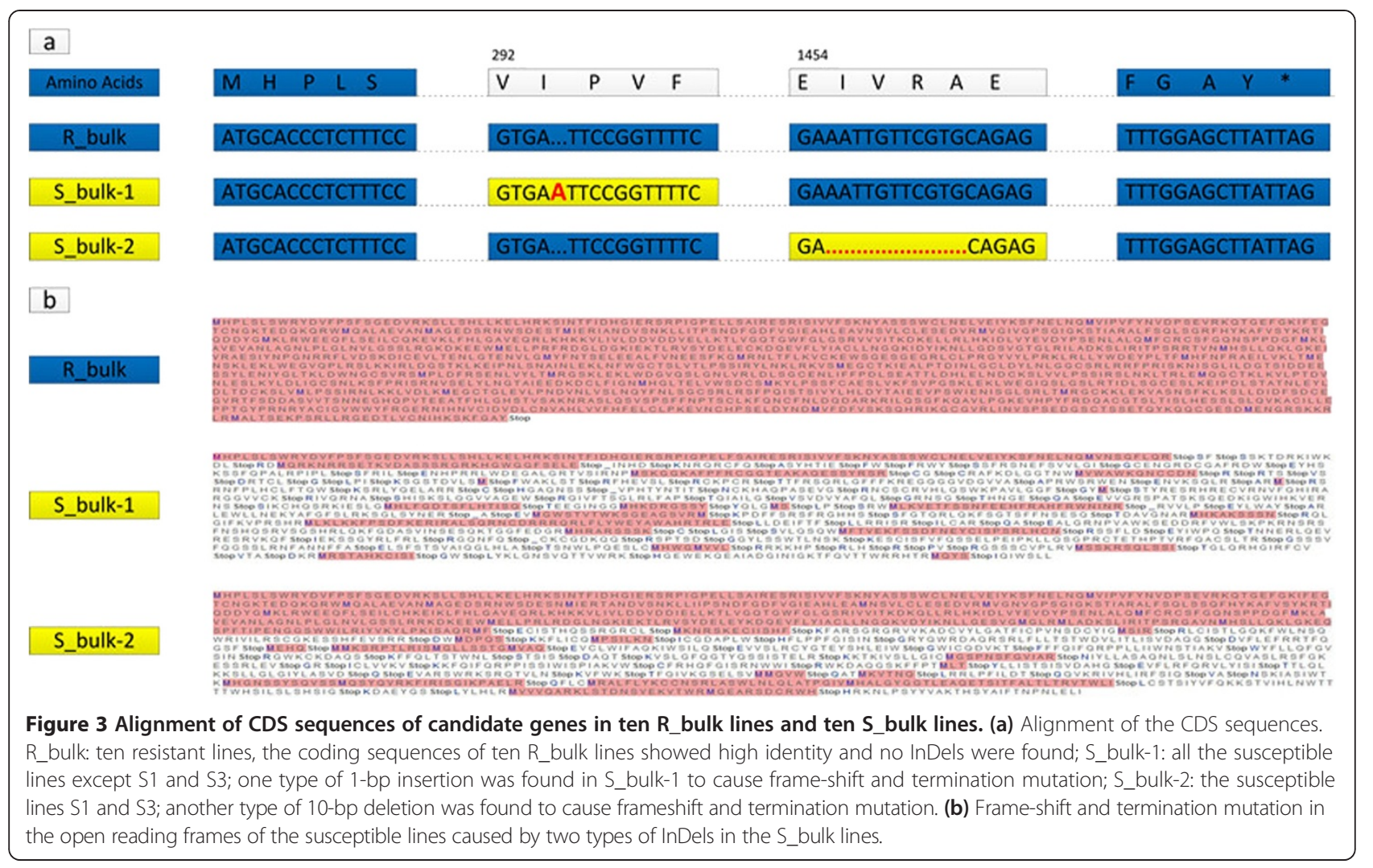

of $<E-10$, using the RFO1, $I-2$ and FOM-2 sequences as the queries. No matches to these sequences were found in the B. oleracea genome. Thus, we can infer that FOC1 may be a novel $\mathrm{R}$ gene. The results of our study showed that the candidate gene for FOC1 was a putative TIRNBS-LRR type $\mathrm{R}$ gene. The cDNA sequences of the ten resistant and ten susceptible lines revealed two types of InDel mutations in the cDNA of the susceptible lines that resulted in frameshifts and translation termination. Further sequence analysis of the two InDel loci from 80 lines confirmed the two types of InDels. Thus, we inferred that the re-modeled gene re-Bol037156 was the most likely candidate gene for FOC1. However, further work is needed to verify the function of FOC1 by transformation.

\section{Conclusions}

In this study we fine mapped a novel cabbage resistance gene to Fusarium wilt using one DH population and one

\section{Table $\mathbf{5}$ Primers used for two InDel loci analysis in $\mathbf{8 0}$} lines

\begin{tabular}{lc}
\hline Primers & \multicolumn{1}{c}{ Sequences $\left(\mathbf{5}^{\prime} \mathbf{- 3}\right.$ ) } \\
\hline 1-bp insertion & AATCACTCCTCAGCCATCTT//ATACCAGTTCCGAGAATCCT \\
10-bp deletion & AAGGAGGAGTGGATGGAG//GCTCTGATGTGTTGAAATAC \\
\hline
\end{tabular}

$F_{2}$ population, analyzed the genes that fell into the candidate region and identified the re-modeled gene reBol037156, a putative TIR-NBS-LRR type $\mathrm{R}$ gene as the candidate for the cabbage Fusarium wilt resistance gene FOC1. This work may lay the foundation for markerassisted selection in cabbage resistance breeding as well as for further function analysis of the FOC1 gene.

\section{Methods}

Plant materials and pathogen strain

To generate $F_{1}$ plants, the inbred-line $99-77\left(\mathrm{P}_{1}\right.$, resistant parent) with immunity to CFW was crossed to the highly susceptible line 99-91 ( $\mathrm{P}_{2}$, susceptible parent). An isolated microspore culture of $F_{1}$ flower buds was adopted to obtain $160 \mathrm{DH}$ lines [20]. At the same time, $\mathrm{F}_{1}$ plants were self-pollinated to generate about 4000 individual $\mathrm{F}_{2}$ plants. All the plant materials were provided by the Cabbage and Broccoli Research Group, Institute of Vegetables and Flowers (IVF), Chinese Academy of Agricultural Sciences (CAAS). The entire inoculation test was performed in the greenhouse of the Plant Protection Research Group at IVF, CAAS.

The strain FGL3-6 of F. oxysporum f. sp. conglutinans used in this study was isolated from diseased plants from Yanqing County, Beijing. This strain had been shown to be Race 1 [5]. 


\section{Evaluation of resistance to Fusarium wilt}

For seedling cultivation, the cabbage seeds were sowed in a sterilized substrate (turf: vermiculite: soil =1: 1: 2) and cultivated in a greenhouse until the third leaf stage.

For inoculum preparation, strain FGL3-6 of the pathogen, maintained on potato dextrose agar medium, was incubated in CM medium (complete medium with casein acids hydrolysate $10 \mathrm{~g} / \mathrm{l}$, casein enzyme hydrolysate $10 \mathrm{~g} / \mathrm{l}$, yeast extract $16 \mathrm{~g} / \mathrm{l}$, and lactose $20 \mathrm{~g} / \mathrm{l}$ ) on a rotary shaker $\left(150 \mathrm{r} / \mathrm{min}, 26^{\circ} \mathrm{C}\right)$ for three days. Double-layer gauze was used to filter the mixture and make a conidium suspension. The concentration of the suspension was adjusted to $1 \times 10^{6}$ conidia/ml for inoculation tests using a hemacytometer.

For the inoculation test, the root-dipping method was adopted to evaluate the resistance of the $\mathrm{DH}$ lines as well as the $F_{2}$ individuals $[9,33,34]$. We picked the seedlings, dipped the roots in the conidial suspension for 15 minutes, and then planted them in plastic pots (diameter $\times$ height $=9 \mathrm{~cm} \times 9 \mathrm{~cm}$ ) with sterilized substrate (turf: vermiculite $=1: 2$ ). The seedlings were cultivated in a greenhouse with temperatures of $27-29^{\circ} \mathrm{C}$ in the day and $23-25^{\circ} \mathrm{C}$ at night. The resistance levels of the plants were evaluated after 10 days.

In a previous study, we found that Fusarium resistance in the populations used in our study was controlled by a single dominant gene. Therefore, for disease rating in the present study, a simple two-grade disease rating standard was adopted to classify the $\mathrm{DH}$ lines and $\mathrm{F}_{2}$ individuals as resistant or susceptible. For the DH population, a randomized trial design was adopted with three replications and each replication consisted of 10 plants. Lines or individuals for which the resistance status was unclear were excluded from further analysis. In the inoculation test, the numbers of resistant and susceptible plants in the $\mathrm{DH}$ and $\mathrm{F}_{2}$ populations were counted. Then, $x^{2}$ goodness-of-fit tests were applied to determine whether the segregation ratios fitted a 1:1 ratio in the $\mathrm{DH}$ population and fitted a 3:1 ratio in the $\mathrm{F}_{2}$ population at a significance level of 0.05 .

\section{DNA extraction}

The cetyl trimethyl ammonium bromide (CTAB) method with minor modifications was used to extract total genomic DNA from the young leaves of 20-day-old plants [35]. The concentration of the DNA samples was determined using a NanoDrop ND-100 spectrophotometer (Thermo Fisher Scientific Inc., Wilmington, DE, USA), and then diluted to a working concentration of $40 \mathrm{ng} / \mu \mathrm{l}$ for PCR analysis.

\section{Molecular maker assay}

The reference sequence of $B$. olerecea was retrieved from the Brassica database (BRAD) (http://brassicadb.org) [21] and used for InDel primer design. The DNA of the parental lines was submitted to whole-genome re-sequencing using the sequencing-by-synthesis method. A total of 7.2 $\mathrm{Gb}$ and 7.1 Gb Illumina pair-end reads were generated for the $\mathrm{P}_{1}$ and $\mathrm{P}_{2}$ parental lines respectively. The reference sequence was used as a 'bridge' to detect sequence polymorphisms between the parental lines [36]. To avoid false detection of polymorphisms, multiple-hit reads were filtered out from the dataset and only single-hit reads were used to design primers. In a prior study, we located the resistance gene to a $1.8 \mathrm{cM}$ genomic region [17]. To map the gene more precisely, additional InDel primers were designed on chromosome C06. For all the primers, the generating products was constrained to be 100-200 bp. The length of primer pairs was limited to 19-22 bp. The Tm value was restricted to be $54-56^{\circ} \mathrm{C}$.

The PCR conditions and contents were as follows: each $20 \mu \mathrm{l}$ PCR reaction mixture contained $2 \mu \mathrm{l}$ PCR buffer $\left(10 \times, \mathrm{Mg}^{2+}\right.$ included), $1.6 \mu \mathrm{l}$ dNTP $(2.5 \mathrm{mM}$ each), $0.4 \mu \mathrm{l}$ Taq DNA polymerase $(2.5 \mathrm{U} / \mu \mathrm{l}), 5 \mu \mathrm{l}$ DNA template $(40 \mathrm{ng} / \mu \mathrm{l}), 0.6 \mu \mathrm{l}$ each of forward and reverse primers $(10 \mu \mathrm{M})$, and $9.8 \mu \mathrm{l} \mathrm{ddH}_{2} \mathrm{O}$. The reaction mixture was incubated in a GeneAmp PCR system 9700 (Applied Biosystems, CA, US) and the PCR profile was as follows: initial $5 \mathrm{~min}$ at $94^{\circ} \mathrm{C}$, then 35 cycles, each with $30 \mathrm{~s}$ DNA denaturation at $94^{\circ} \mathrm{C}, 30 \mathrm{~s}$ annealing at $55^{\circ} \mathrm{C}$ and $45 \mathrm{~s}$ extension at $72^{\circ} \mathrm{C}$, and a final extension of $7 \mathrm{~min}$ at $72^{\circ} \mathrm{C}$. The PCR products were separated on an $8 \%(\mathrm{w} / \mathrm{v})$ polyacrylamide gel at $160 \mathrm{~V}$ for $1.5 \mathrm{~h}$ and stained with silver nitrate [37].

\section{Statistical analysis and gene mapping}

In the DH population, for each pair of primers, the allele of an individual that was the same as the corresponding allele in $P_{1}$ (99-77) was scored as 'a', while the allele that was the same as the corresponding allele in $P_{2}$ (99-91) was scored as 'b'. The data was analyzed with JoinMap 4.0 using a minimum logarithm of odds (LOD) score of 4.0 [38]. The Kosambi function was used to convert the recombinant value to genetic distance [39]. In this step, the gene was preliminarily mapped to an interval with two flanking markers.

The flanking markers obtained from the DH population were used to screen the $F_{2}$ population for recombinants. The recombinants were further genotyped using the markers that mapped between the flanking ones. Except for the ' $a$ ' and ' $b$ ' alleles, individuals that had both the alleles from the two parents were scored as ' $h$ '. In this step, we obtained a narrowed candidate interval with new flanking markers.

To identify the probable genes associated with disease resistance, genes that fell into the candidate interval were analyzed based on the annotations for the B. oleracea reference chromosomes from BRAD. 


\section{Analysis of the candidate gene}

To identify which one was the candidate gene, primers were designed and used to amplify the genomic DNA and cDNA of ten resistant cabbage inbred-lines (R_bulk) including the resistant parent $\mathrm{P}_{1}$ and ten susceptible lines (S_bulk) including the susceptible parent $\mathrm{P}_{2}$. The obtained sequences were aligned to detect common differences between the R_bulk and S_bulk lines. Comparative genomics analysis between $B$. rapa and $B$. oleracea in the target region was performed in order to help identify candidate genes. RT-PCR and transcriptome reads (RNA sequencing was adopted to obtain the transcriptome using RNA extracted from the roots of the seedling six days after inoculation) mapping were also conducted to help confirm the candidate gene for FOC1.

\section{Validation of the candidate gene}

To further validate the candidate gene for FOC1, we chose 40 different highly resistant cabbage inbred-lines including the former ten resistant lines to make the R_bulk and 40 highly susceptible lines including the former ten susceptible lines to make the S_bulk. Primers were designed to amplify the mutation loci of the candidate gene in these 80 cabbage inbred lines. The obtained sequences were aligned to detect common variation between the R_bulk and S_bulk lines.

\section{Availability of supporting data}

The reference genome sequence of $B$. oleracea was retrieved from BRAD, the genomic database for B. oleracea (available at http://brassicadb.org). As one of the data owners, we confirm that we have full permission to use the genome sequence of Brassica oleracea, including downloading and using it for the purposes of this study (i.e. candidate gene identification and characterization). The use of the reference genome for these purposes was performed with the agreement of all the data owners.

\section{Abbreviations}

FOC1: Resistance gene to Race 1 of Fusarium oxysporum f. sp. conglutinans; CFW: Cabbage Fusarium wilt; DH: Double haploid; CDS: Protein coding sequence; InDel: Insertion/deletion; MAS: Marker-assisted selection; SNP: Single nucleotide polymorphism; PCR: Polymerase chain reaction; R gene: Resistant gene; S gene: Susceptible gene; SSR: Simple sequence repeat; NBS: Nucleotide binding site; TIR: Toll-interleukin receptor; LRR: Leucine-rich repeat; RT-PCR: Reverse transcription-PCR; CC: Coiled coil.

\section{Competing interests}

The authors declare that they have no competing interests.

\section{Authors' contributions}

$\mathrm{HL}$ developed the $\mathrm{DH}$ and $\mathrm{F}_{2}$ populations, and wrote and revised the manuscript. HL and QW isolated the samples, performed the marker assays, and analyzed the marker data. XW, JK, ZF, and LY conceived the idea and critically reviewed the manuscript. $B L$ and $J L$ analyzed the sequencing and re-sequencing data and designed the InDel primers. XW, JK, MZ, YZ, YY, and $\mathrm{BX}$ coordinated and designed the study. All the authors have read and approved the final manuscript.

\section{Acknowledgments}

This work was financially supported by grants from the National High Technology Research and Development Program of China (863 Program, 2012AA100101, 2012AA100103, 2012AA100102, 2012AA100105), the Key Projects in the National Science \& Technology Pillar Program during the Twelfth Five-Year Plan Period (2012BAD02B01, 2013BAD01B04), the earmarked fund for the Modern Agro-Industry Technology Research System, China (nycytx-35-gw01), the Major State Basic Research Development Program (973 Program, 2012CB113906) and the National Natural Science Foundation of China $(31171958,31272180,31272003)$. The work reported here was carried out in the Key Laboratory of Biology and Genetic Improvement of Horticultural Crops, Ministry of Agriculture, Beijing 100081, China.

Received: 16 October 2013 Accepted: 26 November 2014

Published: 12 December 2014

\section{References}

1. Smith EF: The fungus infection of agricultural soils in the United States. Sci Am Sup 1899, 48:19981-19982.

2. Nomura Y, Kato K, Takeuchi S: Studies on the method of early selection of the resistance of cabbage to the yellows disease. Jpn Cent Agric Exp Rep 1976, 24:141-182.

3. Li M, Zhang T, Li X, Yan H: Fusarium wilt disease on curcifer vegetable and its pathogenic identification. Plant Prot 2003, 29(6):44-45.

4. Zhang Y, Zheng J, Xie B, Li J, Wu X, Shi Y, Ma Y: Identification on pathogen of cabbage wilt disease. Acta Phytopathologica Sinaca 2008, 38(4):337-345.

5. Lv H, Fang Z, Yang L, Xie B, Liu Y, Zhuang M, Zhang Y, Yang Y: Research on screening of resistant resources to Fusarium wilt and inheritance of the resistant gene in cabbage. Acta Horticulturae Sinica 2011, 38(5):875-885.

6. Arden S: Fusarium yellows of cabbage and related crops. Vegetable Crops 1979, 730:730-731.

7. Keinath AP, Farnham MW, Smith P: Reactions of 26 cultivars of Brassica oleracea to yellows in naturally infested soil. Biol Cult Tests 1998, 13:155.

8. Farnham MW, Keinath AP, Smith JP: Characterization of Fusarium yellows resistance in collard. Plant Dis 2001, 85(8):890-894.

9. Booth C: The Genus Fusarium. UK(Kew) and US(Surrey): Commonwealth Mycological Institute; 1971.

10. Armstrong GM, Armstrong JK: Formae Speciales and Races of Fusarium Oxysporum Causing Wilt Diseases. University Park PA: The Pennsylvania State University Press; 1981.

11. Walker JC: Inheritance of Fusarium resistance in cabbage. J Agric Res 1930, 40:721-745

12. Walker JC, Hooker WJ: Plant nutrition in relation to disease development. I. Cabbage yellows. Am J Bot 1945, 32:314-320.

13. Ramirez-Villupadua J, Endo RM, Bosland P, Williams PH: A new race of Fusarium oxysporum $\mathrm{f}$. sp. conglutinans that attacks cabbage with type $A$ resistance. Plant Dis 1985, 69(7):612-613.

14. Bosland PW, Williams PH, Morrison RH: Influence of Soil Temperature on the Expression of Yellows and Wilt of Crucifers by Fusarium oxysporum. Plant Dis 1988, 72(9):777-780.

15. Jiang M, Zhao Y, Xie J, Tian R, Chen Y, Kang J: Development of a SCAR marker for Fusarium Wilt Resistance in Cabbage. Sci Agric Sinica 2011, 44(14):3053-3059.

16. Pu Z, Motoki S, Zhang Y, Tomohiko N, Takeshi H, Hidetaka H, Satoru M, Ryo $\mathrm{F}$, Keiichi $\mathrm{O}$ : Genetic mapping of a fusarium wilt resistance gene in Brassica oleracea. Mol Breed 2012, 30:809-818.

17. Lv $H$, Yang L, Kang J, Wang $Q$, Wang $X$, Fang Z, Liu $Y$, Zhuang $M$, Zhang $Y$, Lin Y, Yang Y, Xie B, Liu B, Liu J: Development of InDel markers linked to Fusarium wilt resistance in cabbage. Mol Breed 2013, 32:961-967.

18. Liu S, Liu Y, Yang X, Tong C, Edwards D, Parkin IA, Zhao M, Ma J, Yu J, Huang S, Wang X, Wang J, Lu K, Fang Z, Bancroft I, Yang TJ, Hu Q, Wang X, Yue Z, Li H, Yang L, Wu J, Zhou Q, Wang W, King GJ, Pires JC, Lu C, Wu Z, Sampath $P$, Wang $Z$, et al: The Brassica oleracea genome reveals the asymmetrical evolution of polyploid genomes. Nat Commun 2014, 5:3930. doi:10.1038/ncomms4930.

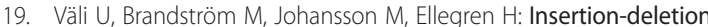
polymorphisms (indels) as genetic markers in natural populations. BMC Genet 2008, 9:8.

20. Takahata $Y$, Keller WA: High frequency embryogenesis and plant regeneration in isolated microspore culture of Brassica oleracea $\mathrm{L}$. Plant Sci 1991, 74:235-242. 
21. The BRAD Database. [http://brassicadb.org]

22. Jeffery $L D$, Jonathan DG: Plant pathogens and integrated defense responses to infection. Nature 2001, 411:826-833.

23. The NCBI BLAST Tool. [http://blast.ncbi.nlm.nih.gov]

24. The Softberry FGENSH Program. [http://linux1.softberry.com/berry.phtml]

25. The Pfam Protein Families Database. [http://pfam.sanger.ac.uk/]

26. Hulbert SH, Webb CA, Smith SM, Sun Q: Resistance gene complexes: evolution and utilization. Annu Rev Phytopathol 2001, 39:285-312.

27. Liu JL, Liu XL, Dai LY, Wang GL: Recent progress in elucidating the structure, function and evolution of disease resistance genes in plants. J Genet Genomics 2007, 34:765-776

28. Steve W, Dinesh-Kumar SP, Doil C, Reinhard H, Catherine C, Barbara B: The product of the tobacco mosaic virus resistance gene Al: Similarity to Toll and the Interleukin-1 Receptor. Cell 1994, 78:1101-1115.

29. Dinesh-Kumar SP, Wai-hong T, Barbara JB: Structure-function analysis of the tobacco mosaic virus resistance gene $N$. Proc Natl Acad Sci U S A 2000, 97:14789-14794.

30. Ori N, Eshed Y, Paran I, Presting G, Aviv D, Tanksley S, Zamir D, Fluhr R: The $I 2 C$ family from the wilt disease resistance locus $I 2$ belongs to the nucleotide binding, leucine-rich repeat superfamily of plant resistance genes. Plant Cell 1997, 9:521-532.

31. Tarek J, Joseph JK, Shelly JN, Claude ET, Ralph AD: The fusarium wilt resistance locus Fom-2 of melon contains a single resistance gene with complex features. Plant J 2004, 39:283-297.

32. Diener AC, Ausubel FM: Resistance to Fusarium oxysporum 1, a dominant arabidopsis disease-resistance gene, is not race specific. Genetics 2005, 171:305-321.

33. Zhang Y, Zheng J, Wu X, Shi Y, Gu P, Li J: Investigation of occurrence and damage of cabbage wilt disease in Yanqing County of Beijing. Chin Agric Sci Bull 2007, 23(5):315-320.

34. Yang $Y, L v H$, Yang $C$, Gong $H$, Yang L, Xie B: The methods for evaluation of cabbage resistance to Fusarium wilt and screen the sources at the seedling stage. Acta Phytophylacica Sinica 2011, 38(5):425-431.

35. Murray $\mathrm{HG}$, Thompson WF: Rapid isolation of high molecular weight DNA. Nucleic Acids Res 1980, 8:4321-4325.

36. Liu B, Wang Y, Zhai W, Deng J, Wang H, Cui Y, Cheng F, Wang X, Wu J: Development of InDel markers for Brassica rapa based on whole-genome re-sequencing. Theor Appl Genet 2013, 126:231-239.

37. Brant JB, Gustavo CA, Peter MG: Fast and Sensitive Silver Staining of DNA in Polyacrylamide Gels. Anal Biochem 1991, 196:80-83.

38. Van Ooijen JW: JoinMap 4, Software for the Calculation of Genetic Linkage Maps in Experimental Populations. Netherlands: Kyazma BV, Wageningen; 2006.

39. Kosambi DD: The estimation of map distances from recombination values. Annu Eugene 1944, 12:172-175.

doi:10.1186/1471-2164-15-1094

Cite this article as: Lv et al:: Mapping and analysis of a novel candidate Fusarium wilt resistance gene FOC1 in Brassica oleracea. BMC Genomics 2014 15:1094.

\section{Submit your next manuscript to BioMed Central and take full advantage of:}

- Convenient online submission

- Thorough peer review

- No space constraints or color figure charges

- Immediate publication on acceptance

- Inclusion in PubMed, CAS, Scopus and Google Scholar

- Research which is freely available for redistribution 